\title{
The Impact of Comorbidities on the Efficacy of Percutaneous Nephrolitotomy (PCNL) in Elderly Patients
}

\author{
Todea-Moga Ciprian*, Boja Radu, Porav-Hodade Daniel, Maier Adrian, Vida Oliver, Mártha Orsolya \\ University of Medicine and Pharmacy Tirgu Mures, Clinic of Urology
}

Introduction: The objective of this study is to evaluate the efficacy and safety of PCNL as a method of treatment of renal stones in elderly patients.

Material and method: This was a retrospective study conducted over a period of 5 years in the Clinic of Urology, where we analyzed the surgical protocols and case reports of 56 patients who underwent PCNL intervention.

Results: The incidence of urolithiasis was higher in females $69.6 \%(n=39)$ than in males $30.4 \%(n=17)$. Comorbidities included hypertension (48.2\%), chronic ischemic cardiopathy (28.6\%), chronic cardiac failure (16.1\%), type II diabetes (17.9\%), obesity (39.3\%), chronic renal failure (8.9\%), chronic or recurrent urinary tract infections (30.4\%), history of kidney stones (21.4\%), solitary kidney surgery (1.8\%), renal malformation (horseshoe kidney and renal incomplete duplication) (3.6\%), urethral stricture (3.6\%). Nine patients had a duble "J" catheter inserted on admission. The group of male patients presented prostate hyperplasia in 35.3\% of the cases and prostate carcinoma in $5.9 \%$ of the cases.

Conclusions: PCNL is an effective and safe treatment of kidney stones in elderly patients, with a stone- free rate increased despite existing comorbidities. The presence of comorbidities requires careful preoperative evaluation. PCNL in elderly patient has similar results to those seen in younger patients.

Keywords: comorbidities, lithiasis, PCNL, renal stones

Received: 23 September 2015 / Accepted: 28 November 2015

\section{Introduction}

Urolithiasis is a common urological condition, ranking the 3rd place as incidence, after urinary tract infections and prostate pathology. Prevalence of urolithiasis varies from $2-20 \%$ worldwide [1], while in Romania there are about 100 cases $/ 100,000$ inhabitants.

Urinary lithiasis is frequent in elderly patients due to metabolic disorders and ageing, and requires prompt treatment as it can have severe effects on renal function $[1,2]$.

When kidney stones are large and can not be spontaneously eliminated, surgical removal is required, which can be achieved by non-invasive methods as lithotripsy extracorporeal shock wave (ESWL), or by minimally invasive methods such as percutaneous nephrolitotomy (PCNL) or ureteroscopy (URS), techniques that replace the classic surgery.

PCNL is a minimally invasive surgical procedure described since 1976, when Fernstrom and conducted the first percutaneous nephrolitotomy [3-5]. Previous studies have shown that the PCNL determines a high rate of stone free cases and is associated with lower morbidity, length of hospital operating time and lower with earlier return to daily activities [6-8].

The objective of this study is to evaluate the efficacy and safety of percutaneous nephrolitotomy as a method of treatment of renal stones in elderly patients.

\section{Material and method}

This paper is a retrospective study conducted over a period of 5 years, from January 2010 to December 2014 in the Clinic of Urology, County Hospital Targu Mures, where we analyzed the surgical protocols and case reports of the patients with orolithiasis. In this period were treated and followed-up 56 patients diagnosed with kidney stones who underwent percutaneous nephrolitotomy intervention.

The inclusion criteria were: elderly patients more than 70 years of age, hospitalized in the period 01.01.2010 31.12.2014 with the diagnosis of renal stones, undergoing percutaneous nephrolitotomy.

We followed the characteristics of the patients, the mean duration of the intervention, the average length of hospitalization, rate of stone free, transfusion and complications.

The diagnosis of kidney stones was established based on renal ultrasound, renal and bladder simple radiography, intravenous urography or intravenous CT scan. These premises were used in specifying the number of urinary calculi and the possible lesions of the upper urinary tract. Preoperative were performed laboratory examinations: complete blood count, kidney function tests, coagulation tests, blood sugar, blood ionogram, summary and urinary sediment, urine culture, electrocardiogram.

Those who were diagnosed with urinary infection at admission received preoperative antibiotic treatment according to their sensitivity. In patients were treated with aspirin for other diseases the treatment was stopped 7-10 days 
before surgery. All existing comorbidities were diagnosed and treated before surgery.

\section{The operative technique}

The standard nephrolitotomy procedure was performed using percutaneous route. The intervention was performed under epidural or spinal anesthesia.

Statistical analysis of data was performed using SPSS (Statistical Package for Social Sciences, version 20, Chicago, IL, USA). Descriptive analysis of data was used: data were included in frequency tables, as number and percentage, then the graphics were made of "sectoral" (pie) and type "columns". Chi-square test was used to check whether there is a significant association between two variables statistically. Significance was determined by materiality $\mathrm{p}=0.05$.

\section{Results}

Most cases of kidney stones treated by PCNL in the studied period: January 2010-December 2014 in Clinic of Urology, Mures County Hospital were recorded in 2012, when $26.8 \%(\mathrm{n}=15)$ of the cases were enocuntered, followed by $25 \%$ in $2014(\mathrm{n}=14), 19.6 \%$ in $2011(\mathrm{n}=11), 17.9 \%$ in $2013(\mathrm{n}=10)$ and $10.7 \%$ in $2010(\mathrm{n}=6)$ (Figure 1).

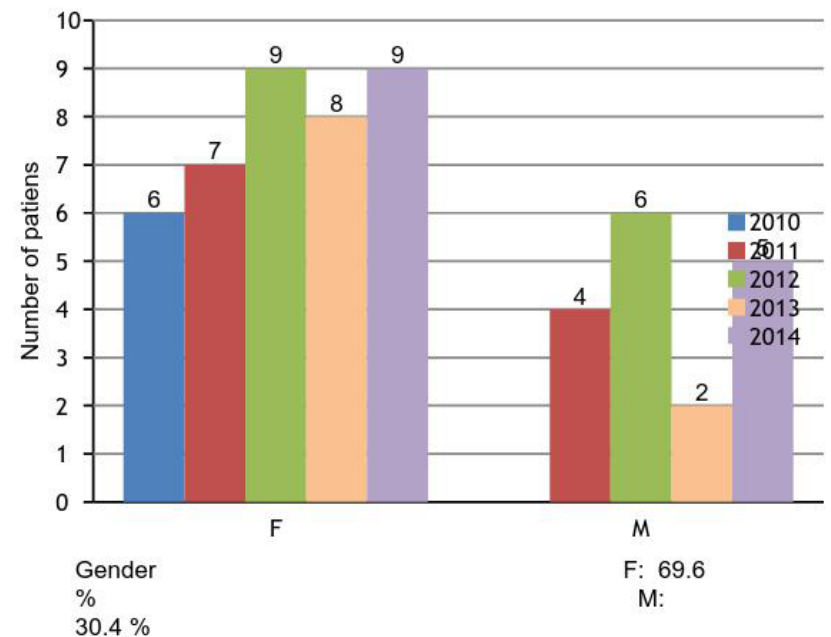

Fig. 1. The distribution of cases by year and gender

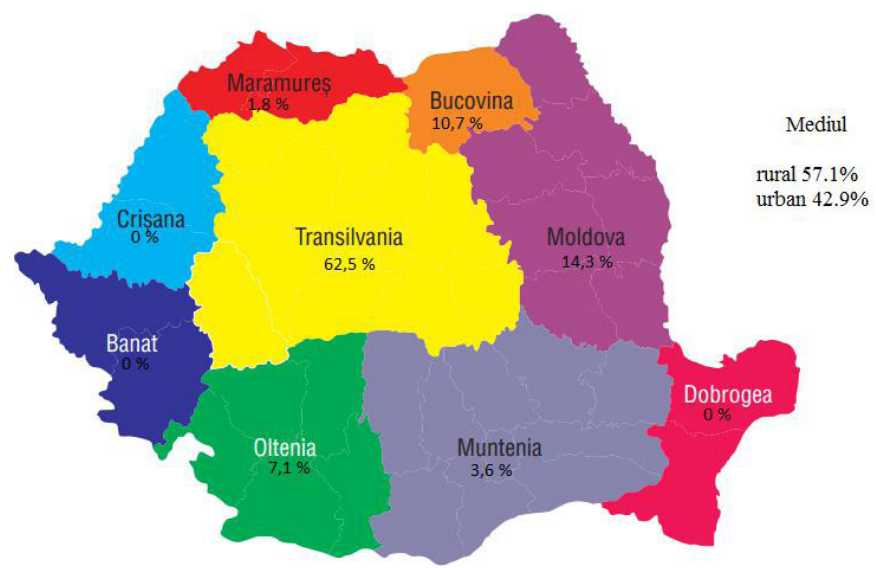

Fig. 2". The geographic distribution of cases with kidney stones
Following the distribution according to geograohic region, we noticed that in more than half of the cases (62.5\%) patients came from Transilvania, followed by Moldova (14.3\%) and Bucovina (10.7\%), while most of the patients came from rural areas $57.1 \%$ (Figure 2).

The age of the 56 patients included in the study ranged between 70 and 84 years, with a mean of 73.9 years, most of them in the 7th decade of life $92.9 \%(\mathrm{n}=52)$ (Table I).

The incidence of urolithiasis was higher in females $69.6 \%(\mathrm{n}=39)$ than in males $30.4 \%(\mathrm{n}=17)$.

Regarding the location of the renal stones, an equal distribution was recorded between the right kidney (46.4\%, $\mathrm{n}=26$ in each), while 4 patients $(7.2 \%)$ had bilateral stones. Location of stones was predominantly in the renal pelvis, encountered in $60.7 \%$ of cases $(n=34)$, followed by coraliform stones $16.1 \%(n=9)$, inferior calycs $5.4 \%(n=3)$, lumbar ureter $5.4 \%(\mathrm{n}=3)$, renal pelvis and inferior calycs, $3.6 \%(\mathrm{n}=2)$, under junction urether $3.6 \%(\mathrm{n}=2)$, multiple lithiasis 3.6\% ( $\mathrm{n}=2)$, medium and inferior calics stones, $18 \%(\mathrm{n}=1)$ (Figure 3). Coraliform stones were type A $(\mathrm{n}=4)$, type $\mathrm{C}(\mathrm{n}=4)$ and $\mathrm{D}(\mathrm{n}=1)$ (Figure 4). In four patients remaining stones were recorded after ESWL or Ureteroscopy.

Twelve patients $(21.4 \%)$ had preoperative urinary tract infection (UTI), 6 with mixed pathogenic flora, 4 with E. coli, one patient with Enterobacter and one with Klebsiella (Figure 5). After the clinical examination $96.4 \%$ of patients $(n=54)$ reported back pain and $85.7 \%$ hematuria $(n=48)$.

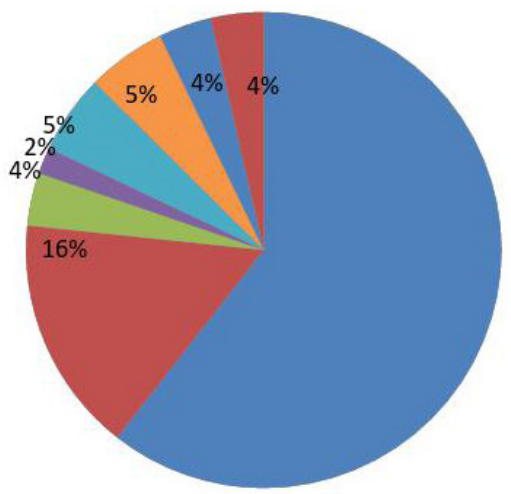

Erenal pelvis

coraliform stone

apelvis and inferior calics

medium and inferior

calics

inferior calics

lumbar urether

Euretheral jonction

multiple lithiasis

Fig. 3. The location of the stones

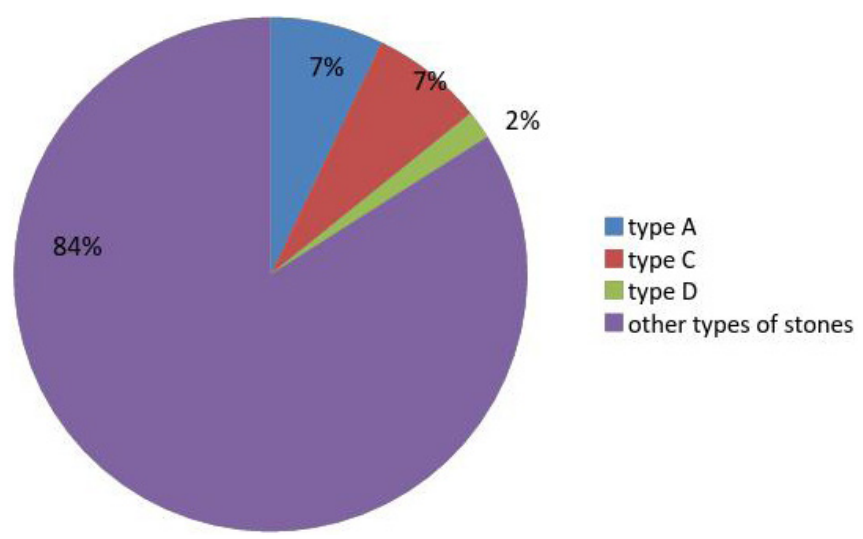

Fig. 4. The type of coraliform stones 
Table I. The distribution of the patients' age

\begin{tabular}{lccccccccccccccccccc}
\hline Age of the patients & 70 & 71 & 72 & 73 & 74 & 75 & 76 & 77 & 78 & 79 & 80 & 81 & 82 & 83 & 0 & 1 & 1 & 2 & 0 \\
\hline $\begin{array}{l}\text { Number of patients } \\
\text { Total }\end{array}$ & 9 & 8 & 7 & 9 & 0 & 5 & 4 & 5 & 5 & 0 & 0 \\
\hline
\end{tabular}

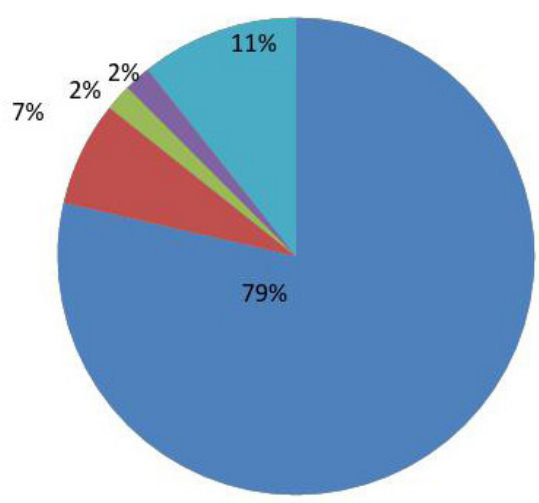

without UTI

E.Coli

Enterobacter

Klebsiella

Mixed

Fig. 5. Preoperator UTI-pathological germs

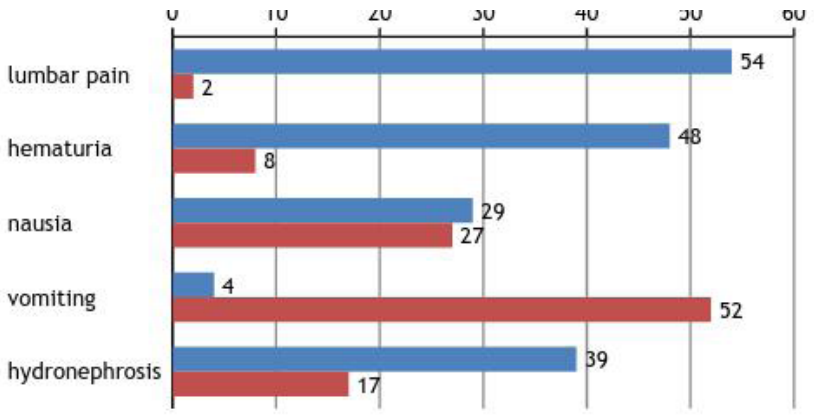

Fig. 6. Clinical aspects

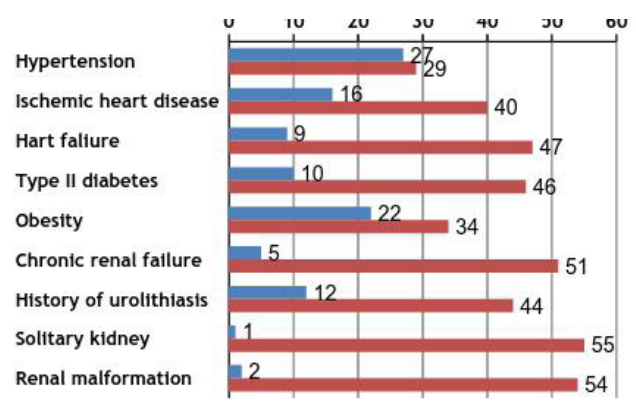

Fig. 7. Comorbidities

Patients also complained about nausea 51.8\% $(\mathrm{n}=29)$ and vomiting $7.1 \%(n=4)$, while ultrasound examination revealed the presence of hydronephrosis in $69.6 \%$ of cases $(\mathrm{n}=39)$ (Figure 6).

Present comorbidities included hypertension (48.2\%), cronic ischemic cardiopathy $(28.6 \%)$, cronic cardiac failure $(16.1 \%)$, diabetes type II (17.9\%), obesity (39.3\%), chronic renal failure $(8.9 \%)$, chronic or recurrent urinary tract infections (30.4\%), history of kidney stones (21.4\%),

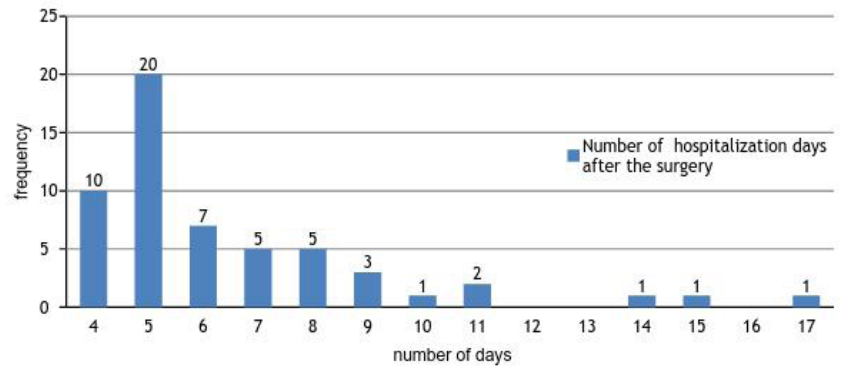

Fig. 8. Number of hospitalization days after the surgery

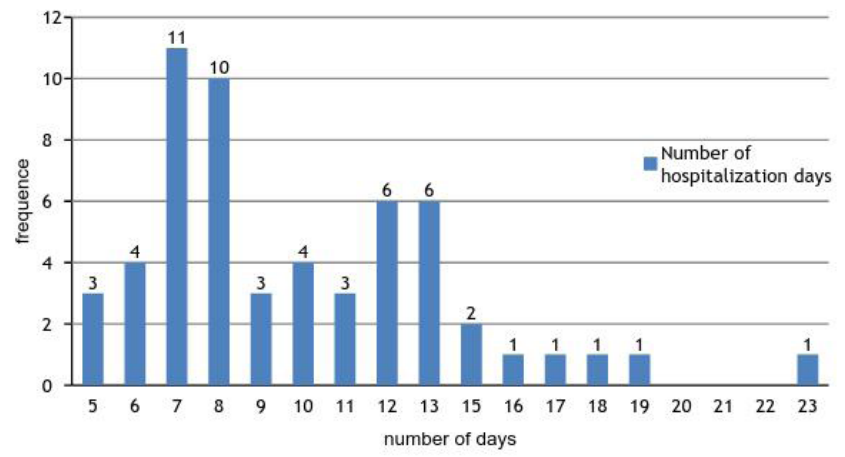

Fig. 9. Number of hospitalization days

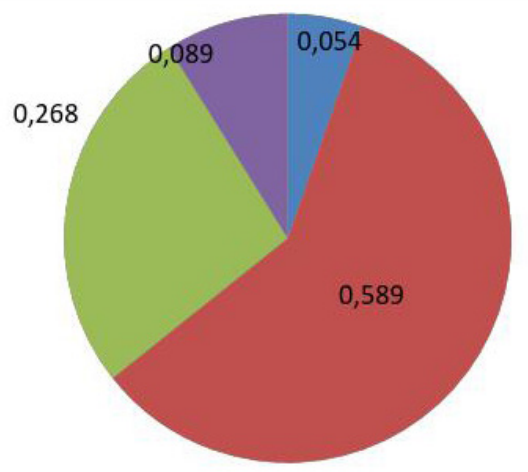

5 days

7 days

10 days

14 days

Fig. 10. Antibiotic therapy

solitary kidney surgery (1.8\%), renal malformation (horseshoe kidney and renal incomplete duplication) (3.6\%), and urethral stricture (3.6\%). Nine patients had duble "J" catheter inserted on admission. Prostate hyperplasia was present in $35.3 \%$ of the male patients, and prostate carcinoma in $5.9 \%$ of them (Figure 7 ).

Most the patients $(89.3 \%, \mathrm{n}=50)$ required one session of PCNL to extract the renal stones, and for $10.7 \%$ of them $(\mathrm{n}=6)$ two surgical interventions were required. In one 
case the fragmentation and the extraction of the stone by PCNL failed, and other methods were required to remove the stones.

The average period of hospitalization was $9.8 \pm 3.6$ days and the average postoperative period of hospitalization was $6.4 \pm 2.8$ days (Figure 8 and 9). All patients received postoperative antibiotic treatment, with a mean duration of 8.3 days (Figure 10). In 7 cases a postoperative urinary tract infection was identified, 4 with mixed pathogenic flora, 2 with E. coli and one with Pseudomonas aeruginosa. One patient presented signs of peritoneal irritation, therefore a diagnostic laparoscopy with peritoneal lavage and drainage were performed, with a favorable evolution. No patients required blood transfusion and there was no case of death. The stone free rate following intervention was $82 \%$.

\section{Discussions}

Percutaneous nephrolitotomy in treatment of urinary lithiasis is a safe and effective procedure in patients over 70 years of age. The postoperative results are not influenced by age or presence of comorbidities.

Surgical treatment of urinary lithiasis follows the principle of minimum invasiveness and morbidity.

Elderly patients have multiple comorbidities, which must be known and controlled, but as it was shown from other studies, they do not influence the results and success of percutaneous nephrolithototmy.

Anagnouston and colleagues [9] believe that the comorbidities associated with old age should not be a concern when the surgeon has the necessary experience.

In our study, patients were aged between 70-84 years, but apparently this did not influence the results of percutaneous nephrolithotomy.

Unlike other studies where most patients were males [10-13], in our work predominated the female patients with $69.6 \%$. However, in another study female patients with renal stones were also majority, representing $61 \%$ of the cases [14].

Other studies on kidney stones in the elderly have reported similar results in terms of the rate of stone-free, period of hospitalization after the surgery or the rate of occurrence of complications and that the PCNL is a efficient method of treatment in renal lithiasis, even in the cases with multiple comorbidities or coraliform calculi $[16,17]$.

Soucy F. et al [13] in his study confirms that PCNL offers safety and efficacy in the treatment of coraliform stones, with similar outcomes for ordinary stones, with smaller sizes.

We had 9 patients with coraliform stones who presented a favorable evolution after surgery.
Doré et al, in a retrospective study comprising $210 \mathrm{pa}-$ tients aged over 70 years, concluded that a history of kidney stones and diabetes influences the rate of stone-free, but other comorbidities, body mass index or age does not have influence [14].

In our work, we did not record any negative impact on patient outcomes in those with a history of urolithiasis $(\mathrm{p}=0.316)$ or diabetes $(\mathrm{p}=0.502)$.

Also in our study, obesity does not seem to influence the results of PCNL $(\mathrm{p}=0.247)$. The same conclusion follows from other works [18-20]. O.Torrencila and colleagues evaluated if the obesity (BMI>30) influence the results of PCNL and found no statistically significant difference regarding the rate of complications in people with normal weight compared to the obese [18]. The period of hospitalization, need for auxiliary procedures and effectiveness of operation are similar in both cases. The differences were only concerning the duration of surgery and radiation exposure, which are higher in people with obesity.

Percutaneous nephrolitotomy intervention is effective in those with solitary kidney, but the cases must be carefully selected [19]. We had only one case of single kidney, whose resulting feedback was favorable $(\mathrm{p}=0.84)$.

The stone-free status was determined using radiography and ultrasound examination after the surgery. Stone- free rate was $82 \%$ in our study. Other studies reported stonefree rate between 36\%-85\% [20]. The higher duration of the intervention increases the risk of complications in the elderly patients. In our study, the average of the surgery duration was 27 to 30 minutes for kidney stones. The average period of hospitalization was $6.4 \pm 2.8$ days. Results of other studies are presented in Table II.

We had 6 patients who required two sessions of PCNL, for various reasons: there were fragments remaining after the first surgery, coraliform stones, or failed to puncture the inferior calyces because of respiratory movements.

\section{Conclusions}

Percutaneous nephrolitotomy is an effective and safe treatment of kidney stones in elderly patients, with a stone-free rate increased despite existing comorbidities. Comorbidities does not represent a risk factor for the evolution of these patinets, however it requires careful preoperative evaluation.

The duration of the procedure and the period of hospitalization were short in our study.

Convalescence and recovery were also short and the patients were able to return to daily activities soon. PCNL in elderly patient has similar results to those seen in younger patients.

Table II. The results of other studies

\begin{tabular}{|c|c|c|c|}
\hline Study & Stone-free rate & Average duration of the surgery(min.) & Average duration of the hospitalization(days) \\
\hline Nakamon T. et al, 2013 & $70,49 \%$ & $52,62 \pm 20,68$ & $5,19 \pm 2,6$ \\
\hline Karami H. et al, 2010 & $85 \%$ & $75 \pm 13,6$ & $3,7 \pm 0,3$ \\
\hline Baris K. et al, 2011 & $36 \%$ & $132,6 \pm 36$ & $3,8 \pm 2,3$ \\
\hline
\end{tabular}




\section{Acknowlegement}

This paper was published under the frame of European Social Found, Human Resources Development Operational Programme 2007-2013, project no. POSDRU/159/1.5/S/133377.

\section{Conflicts of interest}

The authors report no conflicts of interest.

\section{References}

1. Smith LH. The medical aspects of urolithiasis: an overview. JUrol. 1989;141:707.

2. Dursum M, Ozbek E, Otunctemur A, et al. Clinical presentation of urolithiasis in older and younger population. Archivio Italiano di Urologia e Andrologia 2014;86(4):249-252.

3. Duchene DA, Pearle MS. Stone and endourology in older adults. Geriatric Urology by Tomas Lindor Griebling, Ed. Springer, New York 2014;357366

4. McCarthy J-P, Skinner TA., Norman RW, et al. Urolithiasis in the elderly. The Canadian Journal of Urology 2011;18:5717-5720.

5. Vicenti F, Gomes CM, Danilovic A, et al. Percutaneous nephrolithotomy: current concept. Indian Journal of Urology. 2009;25:4-10.

6. Al-Kohlany KM, Shokeir AA. Treatment of complete staghorn stones: a prospective randomized comparison of open surgery versus percutaneous nephrolithotomy. J Urol. 2005;173:469-473.

7. Tiselius HG, Ackermann D, Alken P, et al. Guidelines on urolithiasis. Eur. Urol. 2001;40:362-371.

8. Tugcu V, Su FE, Kalfazade N, et al. Percutaneous nephrolithotomy in patients with previous open stone surgery. Int. Urol. Nephrol. 2008;40:881-884.
9. Anagnostou T, Thompson T, et al. Safety and outcome of percutaneous nephrolithotomy in the elderly: retrospective comparison to a younger patient group. J Endourol. 2008;22:2139-2145.

10. Nakamon T, Kitirattrakarn P, Lojanapiwat B. Outcomes of percutaneous nephrolithotomy: comparison of elderly and younger patients. IBJU 2013;39:692-701.

11. Karami H, Mohsen M, Golshan A, et al. Does age affect outcomes of percutaneous nephrolithotomy? J Urol. 2010;7:17-21.

12. Kuzgunbay B, Turune T, Yaycioglu O, et al. Percutaneous nephrolithotomy for staghorn kidney stones in elderly patients. Int Urol Nephrol. 2011;43:639-643.

13. Soucy F, Ko R, Duvdevani M, et al. Percutaneous nephrolithotomy for staghorn calculi: a single center's experience over 15 years. J Endourol. 2009;23:1669-1373.

14. Dorè $B$, Conort $\mathrm{P}$, Irani J, et al. Percutaneous nephrolithotomy (PCNL) in subjects over the age of 70: a multicenter retrospective study of 210 cases. Prog Urol. 2004;14:1140-1145.

15. Kamphuis GM, Baard J, de la Rosette JJ, et al. Lessons learned from the CROES percutaneous nephrolithotomy global study. World J Urol. 2015;33:223-233.

16. Fuller A, Razvi H, Denstedt JD, et al. The CROES percutaneous nephrolithotomy global study: the influence of body mass index on outcome. J Urol. 2012;188:138-144.

17. Tomaszewski JJ, Smaldone MC, et al. Outcomes of percutaneous nephrolithotomy stratified by body mass index. J Endourol. 2010;24):547550.

18. Torrencila OC, Meza Martinez A, Vicens Morton AS, et al. Obesity in percutaneous nephrolithotomy. Is body mass index really important? J Urol. 2014;84:538-543.

19. Sahin A, Atsü N, Erdem E, et al. Percutaneous nephrolithotomy in patients aged 60 years or older. J Endourol. 2001,15:489-491.

20. Stuard Wolf J. Percutaneous approaches to the upper urinary tract collecting system, in: Kavoussi LR, Novick AC, Partin AW: Urology, 10th ed., Elsevier, 2012;47:1324-1356. 